\title{
Der Komponist als Bühnenfigur in Stefan Herheims Inszenierung von Tschaikowsky: Pique Dame Oder: Ceci n'est pas Tchaïkovski
}

Kadja Grönke

Ein Opernabend beginnt nicht erst mit dem Einsetzen der Musik. Diese Einsicht gilt insbesondere für die Inszenierung von Peter Tschaikowskys Oper Pique Dame (Libretto von Modest und Peter Tschaikowsky, UA St. Petersburg 1890), die der norwegische Regisseur Stefan Herheim ("1970, Oslo) als Koproduktion der Nationale Opera Amsterdam und des Royal Opera House London 2016 in Amsterdam und 2019 in überarbeiteter und zugespitzter Fassung in London auf die Bühne gebracht hat. ${ }^{1}$ Von dem Augenblick an, in dem im Saal die Lichter erlöschen, thematisiert Herheim konsequent, wie das theatrale Handwerk des Regisseurs die widerstreitenden Interessen von künstlerischer Illusion und vermeintlicher Lebenswirklichkeit unter einer übergreifenden Idee zusammenbringt: Zusätzlich zu der musikalischen und szenischen Umsetzung von Tschaikowskys Opernpartitur erzählt er mit den Mitteln dieser Partitur und des Theaters eine weitere, andere Geschichte, die bereits begonnen hat, sobald sich der Vorhang hebt.

Das über den Abend hinweg konstante Einheitsbühnenbild zeigt ein reiches, großbürgerliches Interieur des späten 19. Jahrhunderts mit großer hinterer Fensterwand, davor einen Flügel, Sitzgruppe und rechts einen Kamin, über dem im Halbdunkel ein weibliches Ganzporträt zu erahnen ist. - Es spricht vieles dafür, Tschaikowskys vorletzte Oper, die 1890 in St. Petersburg uraufgeführt wurde, bühnenbildlich, so wie Herheim das tut, in der Zeit ihrer Entstehung zu verorten. Denn von der spielerisch leichten, humorvoll-ironischen literarischen Vorlage, die Alex-

\footnotetext{
Von der Amsterdamer Version ist eine DVD erhältlich (C Major Entertainment $\mathrm{CmbH}, 2018$ ); die überarbeitete Londoner Fassung wurde am 22.01.2019 als Live-Mitschnitt in ausgewählten Kinos gezeigt. Im Folgenden bezieht sich die Beschreibung der Szenenbilder auf den im Kino gesendeten Londoner Mitschnitt. Auf Screenshots muss leider verzichtet werden, da der Leiter der »Business Affairs« des Opernhauses trotz der Zustimmung des kompletten Regieteams die Abdruckerlaubnis verweigert hat.
} 
ander Puschkin 1834 veröffentlichte ${ }^{2}$ hat Tschaikowsky nicht mehr als die drei zentralen Personen übernommen. Die Geschichte um den armen Offizier Hermann, ${ }^{3}$ die alte Gräfin, die angeblich das Geheimnis dreier beim Glücksspiel stets gewinnender Spielkarten kennt, und Lisa, die bei der Gräfin lebt und über die Hermann an das Kartengeheimnis zu gelangen hofft, holt er ganz in seine Gegenwart und macht daraus ein existenzielles Drama über Leidenschaft und fehlgeleitetes, todbringendes Begehren. $\mathrm{Zu}$ diesem Zweck verschärft er den personalen Konflikt. Bei ihm ist Lisa die reiche Enkelin der alten Gräfin und zudem mit dem sie aufrichtig liebenden Fürsten Jelezki verlobt (den Tschaikowsky zur Handlung dazuerfindet). ${ }^{4}$ Aber sie sehnt sich nach dem ihr anfangs noch unbekannten, dämonisch-leidenschaftlichen Hermann. Dieser verzweifelt an ihrer Verlobung und dem Standesunterschied; nur durch Reichtum glaubt er sie für sich gewinnen zu können. Als er der Gräfin dafür das Kartengeheimnis abpressen will, stirbt die alte Frau vor Schreck. Zwar kehrt sie als Geist zurück und nennt ihm unter der Bedingung, Lisa $\mathrm{zu}$ heiraten, drei gewinnbringende Karten. Aber Hermann ist mittlerweile so stark auf Geld und Glücksspiel fixiert, dass er den eigentlichen Auslöser seines Begehrens, die Liebe, vergisst und Lisa dadurch in den Selbstmord treibt. Im Spielsaal gewinnt Hermann tatsächlich zweimal gegen die Bank, beim dritten Mal tritt er gegen seinen Rivalen Jelezki an, zieht die falsche Karte, verliert alles und nimmt sich ebenfalls das Leben.

Die Opernpartitur und ihre von Tschaikowsky bis in wesentliche Teile des Librettos hinein selbstentworfene Gesamtdramaturgie zeigen, welch ein Gespür für wirkungsvolle Bühneneffekte, dramatische Personenkonstellationen und psychologisch komplexe Charaktere der Komponist besaß. Diese zentralen Qualitäten sind eingebunden in die musikalischen Fixpunkte einer Großen Oper mit Chor, Kinderchor und Tanzpantomime, Geistererscheinung, mehrteiligen Arien, musikalisch komplex strukturierten Szenen, leitmotivartigen Verknüpfungen und einer Musik von hochromantischer Leidenschaft.

Herheims Inszenierung tastet all diese Qualitäten des Werks nicht an, unterlegt dem Ganzen aber eine zusätzliche, sehr eigene Lesart, die das Publikum dazu auffordert, sich auf die mannigfachen Zeichen und Binnenverweise von Herheims Erzählwelt und Bildersprache einzulassen. Zudem setzt sie ein gewisses Maß an

2 Erstausgabe: P. [Aleksandr Sergeevič Puškin]: »Pikovaja dama«, in: Biblioteka dlja čtenija [Lesebibliothek], 2 (1834), S. 109-140.

3 Da das Russische kein $>\mathrm{H}<$ kennt, heißt diese Figur bei Puschkin in russifizierter, aber den deutschen Ursprung beibehaltender Form »Germann«, in Tschaikowskys Opernlibretto, gänzlich russifiziert, »German«; die deutschen und englischen Libretti wählen meist die Form »Hermann«.

4 Zu Tschaikowskys Puschkin-Rezeption in dieser Oper siehe z.B. Kadja Crönke: Čajkovskijs Puškin-Opern. Aspekte einer Werke-Einheit (Čajkovskij-Studien 5), Mainz u.a. 2002. 
Vorkenntnissen über den Komponisten der Oper voraus. Im Internet-Blog des Londoner Royal Opera House wurde daraufhin vorgeschlagen: "to be fair the name should be different: something like >Tchaikovksy and the Queen of spades ${ }^{5}{ }^{5}$

Die hier betonte feste Verbindung zwischen dem Komponisten, seinem Werk und dessen Entstehung, wie Herheim sie imaginiert, manifestiert sich bereits in dem vom Regisseur hinzuerfundenen musikfreien Vorspiel, das dem Opernabend voransteht. Im Halbdunkel des Bühnenbildes ist mit dem Rücken zum Publikum in einem Lehnstuhl vorn rechts auf der Bühne ein nachlässig angezogener, entspannt hingeflegelter Mann zunächst mehr erahnbar als erkennbar. Er zieht die Aufmerksamkeit erst in dem Augenblick auf sich, als er mit einem geradezu animalischen Wutschrei einen anderen, großbürgerlich und korrekt gekleideten Mann von sich stößt, der zuvor für das Publikum unsichtbar vor oder zwischen seinen Beinen gekniet hat. Als dieser seine Hand küssen will, wird er erneut brüsk zurückgewiesen: >Mann eins` will keine Zuneigungsbekundungen, sondern Geld. Kaum hat er es erhalten, schlüpft er in eine Uniformjacke und geht. Der Zurückbleibende flüchtet sich an den Flügel. In demselben Augenblick, in dem er ein Notenblatt in die Hand nimmt, setzt aus dem Orchestergraben die Musik zu Pique Dame ein: Der verschmähte Liebende wird zu dem Komponisten Peter Tschaikowsky.

Doch auch ohne Flügel und Notenblatt wäre die Verbindung offenkundig: Der Vergleich mit einer Fotografie Tschaikowskys ${ }^{6}$ aus dem Jahr 1890 (dem Entstehungsjahr der Oper) zeigt deutlich, dass Maske und Kostüm sich eng an historisches Bildmaterial anlehnen - von Frisur und Bart bis hin zur Krawattennadel und Details der Bekleidung. ${ }^{7}$ Die erste Beobachtung zu Stefan Herheims Regiekonzept lautet also: Ganz offensichtlich stellt Herheim in seiner Inszenierung der Oper Pique Dame den Menschen Tschaikowsky zur Entstehungszeit seiner Oper auf die Bühne.

»Absolutely amazing and fresh interpretation of the `Queen of spades«, with unpredictable twists of narrative, smart jokes and modern tragedy of the genius on stage. Sorry that people didn't enjoy it, though to be fair the name should be different: something like >Tchaikovksy and the Queen of spadess. Hope at least that everyone will now decide to go and see the production themselves, instead of reading reviews. « Eintrag mit Autorenkürzel »D. I.« am 22.01.2019, in: Your Reaction: What Did You Think of Tchaikovsky's sThe Queen of Spadess? Audience Responses and Press Reviews of Stefan Herheim's Staging of Tchaikovsky's Ambitious Opera, hg. von Mel Spencer, in: Royal Opera House London: Premierenseite zu »The Queen of Spades«, Abschnitt »Blog«, https://www.roh.org.uk/news/your-reaction-what-did-you-think-of-tchaiko vskys-the-queen-of-spades (abgerufen am 06.09.2019).

6 Vgl. Fotografie 79a in »Photographs«, in: Tchaikovsky Research, http://en.tchaikovsky-research .net/pages/Photographs (abgerufen am 06.09.2019).

7 Die Rolle des Hermann wurde 2016 in Amsterdam von Misha Didyk, 2019 in London von Vladimir Stoyanov gesungen. 
Angesichts von Tschaikowskys Homosexualität, die durch Publikationen wie Alexander Poznanskys Tchaikovsky: The Quest for the Inner Man ${ }^{8}$ oder Valerij Sokolovs Briefe Čajkovskijs ohne Kürzungen ${ }^{9}$ mittlerweile zum gängigen biographischen Wissen dazugehört, drängt sich nahezu zwangsläufig die Frage auf, ob die von Herheim erfundene Eingangsszene den Abschluss einer körperlichen Dienstleistung Tschaikowskys an einem Fremden darstellt, für die Tschaikowsky nicht nur bezahlen, sondern sich auch noch verspotten lassen muss. Herheims Amsterdamer Inszenierung von 2016 erlaubt kaum eine andere Deutung. Die Londoner Version von 2019 lässt dagegen auch andere Interpretationen zu. Vor allem aber bringt sie deutlich mehr Aggression ins Spiel: Tschaikowskys Wunsch nach emotionaler Hingabe, die sich im Handkuss ausdrückt, wird von dem anderen Mann mit brutaler Vehemenz abgewehrt; der Bezahlende muss sich nicht nur auslachen, sondern hier auch als »Verrückten ${ }^{10}$ beschimpfen lassen. - Die Ausgangsbeobachtung verlangt also nach einer Korrektur: Ganz offensichtlich stellt Stefan Herheim nicht den Menschen Tschaikowsky, sondern den Homosexuellen Tschaikowsky auf die Bühne.

Durch sein zugefügtes Vorspiel signalisiert der Regisseur, dass die Oper Pique Dame, deren Aufführung folgt, das Werk eines Homosexuellen in einer homosexualitätsfeindlichen Zeit ist. Dabei handelt es sich nicht um eine Art vorangestelltes Motto, denn die Figur Tschaikowsky bleibt bis zum Ende des Abends auf der Bühne, wird Teil der Szene, ja sogar Teil der Opernhandlung und spielt dadurch auf facettenreiche Weise mit jener Identifikation, die der historische Tschaikowsky mit seinen Bühnenfiguren für sich postuliert hat: »In Wahrheit«, bekannte der Komponist in einem Brief, »war Hermann für mich nicht nur ein Vorwand, diese oder jene Musik zu schreiben, sondern eine wirkliche, lebendige Person, und eine, die mir sehr sympathisch ist. ${ }^{11}$ "Schrecklich geweint, als Hermann seinen

$8 \quad$ Alexander Poznansky: Tchaikovsky: The Quest for the Inner Man, New York u.a. 1991.

9 Valerij Sokolov: »Briefe P. I. Čajkovskijs ohne Kürzungen: Unbekannte Seiten seiner Korrespondenz«, in: Thomas Kohlhase (Hg.): Čajkovskijs Homosexualität und sein Tod: Legenden und Wirklichkeit, sowie weitere Beiträge anderer Autoren (Čajkovskij-Studien 3), Mainz u.a. 1998, S. $137-162$.

10 So wie die vorangestellte Szene zwischen den beiden Männern insgesamt von Herheim der Oper hinzugefügt wurde, so entstammt auch dieser beleidigende Ausruf an ihrem Ende nicht dem Opernlibretto.

11 Brief von Tschaikowsky an seinen Bruder Modest vom 03./15.03.1890 in: Petr Il'ič Čajkovskij: Polnoe sobranie sočninij [Cesamtausgabe], Bd. 15b: Briefe 1890, hg. von Ksenija Davydova und Galina Labutina, Moskau 1977, S. 85-88, hier übersetzt von Kadja Crönke nach dem Wiederabdruck auf Tchaikowsky Research: http://en.tchaikovsky-research.net/pages/Letter_4058 (abgerufen am 06.09.2019). 
Geist aufgab «, ${ }^{12}$ notierte er in seinem Tagebuch. - So gesehen, verlangt die Ausgangsbeobachtung nach einer weiteren Präzisierung. Offenkundig muss sie lauten: In seiner Deutung von Pique Dame stellt Stefan Herheim den Homosexuellen Tschaikowsky als Schöpfer einer Oper auf die Bühne, mit deren Figuren sich der Komponist sympathetisch verbunden fühlt.

Dieser Ansatz ist kennzeichnend für Stefan Herheims Konzept einer Musiktheaterregie, die den künstlerischen Schaffensprozess mit lebendig machen will. Am deutlichsten wurde das bislang in Richard Wagners Meistersingern (Salzburger Festspiele, Premiere am 2. August 2013), deren gesamte Handlung Herheim als Visualisierung dessen inszenierte, was der Schuster und Dichter Hans Sachs im Laufe des Abends erfindet, aufschreibt und mit den übrigen Opernfiguren sofort zur Aufführung bringt - bis hin zu dem nachdrücklichen Regieeinfall, dass der Biedermeierschreibtisch von Sachs zum überdimensionalen Bühnenbild wird, die Figuren also tatsächlich gewissermaßen direkt auf dem Papier ihre Bühnenwirklichkeit erleben.

Für Tschaikowskys Oper geht Herheim noch einen Schritt weiter: Hier ist der Schöpfer nicht nur ein Dichter, sondern ein Komponist, den wir beim Entwerfen und Niederschreiben von Handlung, Text und Partitur seines Musiktheaterwerks Pique Dame erleben. Immer wieder im Verlauf des Abends tritt der BühnenTschaikowsky an den Flügel, wo er sowohl die gerade erklingenden Passagen dirigiert oder mit dem Gänsekiel aufschreibt als auch am Klavier spielend und komponierend die Handlung vorantreibt. Wir erleben also auf der Bühne den Schaffensvorgang und das Erschaffene zugleich, und in Herheims Regie tritt nun beides in eine facettenreiche Wechselwirkung. - Folglich ist eine letzte Schärfung der Ausgangsbeobachtung angebracht, die in voller Länge lauten muss: Stefan Herheim stellt den homosexuellen Künstler Tschaikowsky auf die Bühne und zeigt ihn beim Komponieren einer Oper, in deren Figuren und Handlungen er eigene Erlebnisse, Wahrnehmungen und Emotionen hineinprojiziert und als Bühnenhandlung sichtbar macht. Tschaikowskys inneres Leben und Erleben und die Entstehung seiner Oper werden zum Gegenstand einer mit den Mitteln der Musiktheaterregie erzählten >Musikgeschichte auf der Bühneく.

Wie das bei einem Werk funktionieren kann, in dem es - anders als bei Wagners Meistersingern - eigentlich gar keinen Künstler als Bühnenfigur gibt, darüber lässt Herheim sein Publikum relativ lange im Unklaren. Anfangs wirkt sein Bühnen-Tschaikowsky lediglich wie eine raffiniert erfundene Regiezutat. Sie erwächst aus einer akribischen Re-Lektüre der Oper vor dem Hintergrund von

\footnotetext{
12 Tagebucheintragung Tschaikowskys vom 02./14.03.1890; hier zitiert nach der deutschen Übersetzung von Hans-Joachim Grimm in: Peter Tschaikowski: Die Tagebücher, hg. von Ernst Kuhn, Berlin 1992, S. 326.
} 
ausgewählten biographischen Informationen über den historischen Komponisten. Wenn Herheim beispielsweise eine Blickachse zwischen seinem BühnenTschaikowsky und dem Frauenporträt über dem Kamin herstellt, steht außer Zweifel, dass dieses Gemälde im Kontext der Oper Pique Dame das für die Handlung unverzichtbare Jugendbildnis der alten Gräfin sein muss. Als Vorlage für dieses Gemälde hat Herheims Ausstatter allerdings eine Fotografie von Tschaikowskys Mäzenin Nadeshda von Meck herangezogen, ${ }^{13}$ sodass es auch für diese Bühnenfigur einen >Sitz im Leben des historischen Komponisten gibt.

Solche Bezüge wirken auf den ersten Blick wie bewusst lancierte Authentizitätssignale, die der Inszenierung den Anschein einer historischen Erzählung über den realen Komponisten Tschaikowsky verleihen. Bei Herheim sind sie allerdings stets in sich mehrdeutig und Teil eines facettenreichen Spiels, bei dem der Regisseur auf der Theaterbühne und mit den Mitteln des Theaters Grundsätzliches über das Theater als solches und über unsere Vorstellungen von Kunst und Künstlertum aussagt. Deutlich wird dies beispielsweise, wenn der Bühnen-Tschaikowsky die ersten Operntakte unmittelbar bei ihrem Erklingen mitdirigiert, aufschreibt und nachliest. Dadurch wirkt der Schaffensprozess wie eine spontane Inspiration, bei der intensive Emotionen unmittelbar auf das Papier fließen - ganz so, wie das Opernpublikum es sich bei dem Schöpfer einer derart emotionalen Musik vielleicht auch vorstellt, wie es aber vor allem für das idealisierte romantische Künstlerbild seit dem 19. Jahrhundert zum Stereotyp geworden ist. Wird Tschaikowsky hier zum Idealtypus des romantischen Künstler-Genies stilisiert - oder steht er nur pars pro toto für das Prinzip des schaffenden Künstlers im Allgemeinen?

Dass weder das Tschaikowsky-Klischee noch das romantische Künstlerstereotyp eine Wahrheit für sich beanspruchen, sondern theatrale Mittel sind, die auf ihre Wertigkeit und Relevanz für das Verständnis der Oper befragt werden müssen, zeigt der Gänsekiel, mit dem der Bühnen-Tschaikowsky seine Noten aufschreibt: Als feine ahistorische Brechung verweist er auf das Fiktive, auf das Gemachte und In-Szene-Gesetzte all dessen, was auf der Bühne zu sehen ist. Gleichzeitig wird er zum optischen Leitmotiv, das in der vielleicht extremsten Passage der Inszenierung kulminiert - jener Szene zu Beginn des dritten Akts (5. Bild der Oper), die auch innerhalb der Opernhandlung die extremste, nämlich die fantastischste und irrealste, ja geradezu surreale Episode ist: Als die Stimme der toten Gräfin Hermann die drei magischen Karten verrät, durch die er beim Glücksspiel zu Reichtum gelangen wird, kann der Bühnen-Tschaikowsky die entsprechende Inspiration für diese Musik nur erlangen, indem er sich mit dem Gänsekiel das Blut aus den 
Adern sticht und damit schreibt. Dazu umtanzen ihn manierierte, grotesk verrenkte halbnackte Männergestalten, die mit Schreibfedern gespickt sind wie mit Pfeilen - wobei statt Blut Tinte aus allen Wunden quillt. Eine Seite dieses Assoziationsangebots ist die hochromantische Vorstellung vom leidenden Künstler, der sein Werk unter Schmerzen mit seinem eigenen Blut niederschreibt. Die andere Seite liegt in dem überdeutlichen Verweis auf das Martyrium des Heiligen Sebastian, des Lieblingsheiligen der Homosexuellen: ${ }^{14}$ Sein extremer Schmerz präsentiert sich der ästhetischen Betrachtung; die Verschmelzung von Leid und Schönheit, Leiden und Genießen wird zum Sinnbild homosexuellen Begehrens - ganz ähnlich wie das Leid des Bühnen-Tschaikowsky, der seinem geliebten Hermann den Weg zu Lisa komponiert, in musikalisch-ästhetischen Genuss transformiert wird. - Dass Herheim damit zugleich dem historischen Tschaikowsky ein Leiden an seiner Homosexualität zuschreibt, welches dieser im Kunstwerk sublimiert, ${ }^{15}$ erweist sich als grundlegend für Herheims Tschaikowsky-Deutung. ${ }^{16}$

Auf die Verbindung von Homosexualität, Leid und Todesnähe verweist auch das optische Leitmotiv eines Wasserglases: Von innen heraus beleuchtet und mit einer milchig trüben Flüssigkeit gefüllt, ist es in Herheims Inszenierung geradezu demonstrativ präsent, steht als Requisit bereit oder wird vom Herrenchor genutzt, um dem Bühnen-Tschaikowsky zuzutrinken. Es verweist auf Hypothesen zum Tod des historischen Tschaikowsky, denen zufolge er entweder ein Glas mit CholeraBakterien kontaminierten Wassers getrunken oder mit Hilfe von Gift Selbstmord verübt haben soll, um seine Homosexualität geheim zu halten. ${ }^{17}$ Damit ist das Glas einerseits ein theatrales Authentizitätssignal, das auf die historische Künstlerbiographie verweist, andererseits aber auch zirkelschlüssig ein fiktionaler Beweis für diese Todesmythen: Das Glas wird von Herheim genutzt, weil es möglicherweise mit dem Tod des historischen Tschaikowsky zu tun hat - und weil es als Verweis auf die Biographie verstanden wird, legt es dem Publikum nahe, dass Tschaikowskys realer Tod etwas mit tödlichem Wasser und folglich auch etwas mit dem Leiden an Homosexualität zu tun haben muss. Herheim spielt also nicht nur mit dem

14 Vgl. z.B.: Irene Ulrich: »Der heilige Sebastian: vom christlichen Märtyrer zur homosexuellen Utopie«, in: Elke Pahud de Mortanges und Franziska Metzger (Hg.): Orte und Räume des Religiösen im 19.-21. Jahrhundert, Paderborn 2016, S. 207-222.

15 Auf die Eindimensionalität dieser Deutung kann hier nicht weiter eingegangen werden.

16 Dass Herheim die Feder zugleich als Verweis auf die optische Metapher des Vogels im Käfig nutzt und dieses wichtige Requisit wiederum die Verbindung zu den klingenden MozartAnspielungen in Tschaikowskys Partitur herstellt, ist ein plastisches Beispiel für die Mehrwertigkeit von Herheims Bildersprache.

17 Vgl. Alexander Poznansky: „Čajkovskijs Homosexualität und sein Tod. Legenden und Wirklichkeit«, in: Kohlhase (Hg.), Čajkovskijs Homosexualität und sein Tod, S. 9-135, und Kadja Grönke: »Čajkovskijs Tod - ein kritischer Literaturbericht«, in: ebd., S. 379-403. 
großen romantischen Künstlermythos des 19. Jahrhunderts, sondern auch mit den wesentlichen Stereotypen des Tschaikowsky-Mythos.

Der Männerchor, der dem Bühnen-Tschaikowsky wiederholt mit dem Wasserglas zutrinkt, und die tanzenden St.-Sebastian-Gestalten treten bei Herheim allesamt als Tschaikowsky-Doppelgänger auf. Dieser überdeutliche Verweis auf die Identifikation des Komponisten mit seinen Figuren suggeriert nicht nur, dass alle Gestalten letztlich einem einzigen Kopf entspringen, sondern mag auch eine feine intertextuelle Anspielung sein: Bereits in dem Film An American in Paris ${ }^{18}$ (USA 1951) gibt es eine Szene, in der der fiktive Komponist Adam Cook, gespielt von Oscar Levant, die Aufführung seines eigenen Klavierkonzertes erträumt, bei der er Solist, Dirigent, das komplette Orchester und sogar sein eigenes Publikum ist. Auch Herheims Bühnen-Tschaikowsky ist nicht nur der Komponist der erklingenden Musik, sondern er studiert sie auch ein, inszeniert sie, und wenn er den Mitwirkenden Notenblätter austeilt und sie auf der Bühne zu wirkungsvollen Tableaus arrangiert, bleibt er letztlich auch selbst nicht außen vor. Während der Bühnen-Tschaikowsky in dem von Herheim hinzugefügten Vorspiel als Opfer seiner unerwiderten Emotionen gezeigt wird, entwickelt er sich im Raum seiner Musik also zum Herrscher über die Gestalten, die er nach seinem Willen erfindet und zu denen er eine starke, auch emotionale Bindung aufbaut. Das geht so weit, dass er dem Offizier, der ihn zu Beginn so demütigt, die männliche Hauptrolle seiner allmählich entstehenden Oper zuweist. Den kaltherzig-brutalen käuflichen Geliebten seiner hässlichen Wirklichkeit verwandelt er in den glücklosen, von leidenschaftlicher Liebe und von der Spielsucht besessenen Helden seiner Bühnenwahrheit und führt ihn am Ende in den Tod - vielleicht eine subtile Form der Rache?

Aber ebenso wie die Ausgangsbehauptung zur Gleichsetzung von Bühnenfigur und historischem Tschaikowsky einer mehrfachen Präzisierung bedurfte, so muss auch diese Beobachtung genauer gefasst werden. Denn als Hermann im ersten Bild des ersten Alkts (Nr. 2) zum ersten Mal die Opernbühne betritt, wirkt es keineswegs so, als habe der Bühnen-Tschaikowsky ihn bewusst herbeikomponiert, sondern als sei der Komponist vielmehr erschrocken, ihn wiederzusehen. So ganz scheint es also nicht zu stimmen, dass er hier unumschränkt über seine Bühnengestalten herrscht. Das Aufeinandertreffen der beiden Antagonisten erweist sich als deutlich komplexer.

Unmittelbar vor Hermanns Erscheinen unterhalten seine Freunde sich über ihren Kameraden: »Was für ein seltsamer Mensch er doch ist! Als hätte er mindestens

18 An American in Paris, Regie: Vincente Minelli, USA 1951, Film mit Gene Kelly und Leslie Caron in den Hauptrollen. 
drei Verbrechen auf dem Kerbholz. ${ }^{19}$ Doch er beachtet sie nicht, sondern geht wie in Trance auf den Flügel zu, wo er sich über die Noten beugt. Erst auf die Frage nach seinem Befinden antwortet er verstört: »Ich weiß selbst nicht, was mit mir los ist. [...] Ich liebe! Liebe! « ${ }^{20}$ Geschickt nutzt Herheim die bereits bekannten optischen Versatzstücke, um seine Figuren zwischen dem aktuell komponierten Bühnenspiel und der Erinnerung an das hinzugefügte Vorspiel hin- und herflackern zu lassen: Der Sessel, hinter dem der Bühnen-Tschaikowsky sich bei Hermanns Auftreten erschrocken versteckt, ist derselbe, in dem am Anfang sein käuflicher Geliebter saß. Doch Hermann ist nicht identisch mit dem herzlosen Offizier des Vorspiels: Indem er sich in die Noten vertieft und die vom Bühnen-Tschaikowsky daraufhin rasch entworfene Klage über qualvoll unerfüllbare Liebe singt (sein Arioso »Ich kenne ihren Namen nicht«, 1. Bild, Ende von Nr. 2), wird er Teil der Oper und Produkt der schöpferischen Fantasie des Bühnen-Komponisten.

Allerdings ist Hermanns Kostüm im Unterschied zu dem seiner Kameraden nicht historisierend (also zum Inhalt der in Tschaikowskys Kopf entstehenden Oper passend) im Rokokostil gehalten, sondern korrespondiert mit Tschaikowskys Bekleidung im Schnitt des 19. Jahrhunderts, und er trägt auch keine Perücke. Anders als der Komponist, der zwischenzeitlich analog zu dem Chor zumindest ein falsches Haarteil angelegt hat, begibt Hermann sich also nicht in die Aufführung hinein, sondern bleibt ein Fremdkörper (so wie er auch in Tschaikowsky Originallibretto, analog zu Puschkins Erzählung, immer wieder als fremd und befremdlich charakterisiert wird). Auf diese Weise bleibt die Vorgeschichte zwischen den beiden Männern optisch präsent und überformt schließlich die Bühnenhandlung. Zwar belässt Herheim das Libretto unangetastet, doch er verleiht ihm mit Hilfe von Gesten und Körpersprache eine zweite Bedeutungsebene: Als Hermann auf die Frage seiner Freunde »Glaubst du denn nicht, dass deine Angebetete deine Liebe erwidert?« die dramatische Schlussfolgerung zieht, »Wenn ich darauf nicht hoffen könnte, bliebe mir nur zu sterben ${ }^{21}{ }^{21}$ richtet er sich mit Blicken, Gesten und Bewegungen direkt an den Bühnen-Tschaikowsky und zeigt bei dem Wort »sterben« mit dem Finger auf ihn. Diese simple Geste bewirkt eine Gleichsetzung von Hermanns gesellschaftlich unmöglicher Liebe zu Lisa mit Tschaikowskys unmöglicher - weil zu seiner Zeit und in seinem Land ungesetzlichen - Liebe zu Männern. Wie das fatale Glas Wasser ist auch diese Geste ein deutlicher >Fingerzeig ‘ auf die Verknüpfung von Homosexualität und Tod. ${ }^{22}$

19 Vgl. Petr Il'ič Čajkovskij: Polnoe sobranie sočninij [Gesamtausgabe], Bd. 9: Pikovaja Dama, Partitur, hg. von Anatolij Dmitriev, Moskau 1950, S. 59. Die zitierten Auszüge aus dem Libretto von Peter und Modest Tschaikowsky sind aus dem Russischen übersetzt von Kadja Grönke.

20 Ebd., S. 64.

21 Ebd., S. 99f. und S. 101-104.

22 Ähnliche optische Umdeutungen des Librettotexts beobachtet auch Philip Ross Bullock in seiner Rezension der Londoner Aufführung (»The Authority of the Author and the Despo- 
Das Hin- und Herflackern zwischen unterschiedlichen Facetten von Rollen und Personen, das Herheim immer intensiver in Szene setzt, lässt den BühnenTschaikowsky nach und nach die Kontrolle über seine Geschöpfe verlieren. Er wird von seinen Operngestalten mehr und mehr als Mitspieler wahrgenommen und in das Bühnengeschehen hineingezogen. Kaum hat Hermann ihn gewissermaßen vor aller Augen und Ohren als Homosexuellen geoutet, zwingen ihn die anderen Bühnenfiguren (fast ironisch) in die Rolle des glücklich heterosexuell verlobten Fürsten Jelezki. Auf die Frage »Gratuliere Fürst, man sagt, du seist verlobt?« antwortet er: »Ja, ein lichter Engel hat sich mit mir verbunden. ${ }^{23}$

Damit wird deutlich, dass die Frage nach der Gestaltungsmacht des Komponisten weitaus mehr Facetten besitzt, als es zunächst den Anschein hat: Der BühnenTschaikowsky entwickelt sich in der von ihm komponierten Musik zwar zum aktiv Handelnden. Aber sein Versuch, die Figuren seiner Oper nach seinem Willen zu gestalten, scheitert an den unterschiedlichen, einander überlagernden und durchdringenden Wahrnehmungs- und Bedeutungsebenen. Bei Hermanns erstem Auftritt im Kreis seiner Kameraden trifft der homosexuelle (gewissermaßen >reale ) Bühnen-Tschaikowsky auf seine (imaginäre) Bühnenfigur Hermann. Diese nimmt jedoch nach und nach Züge des (>realen`) Offiziers aus dem Vorspiel an und bedroht damit die Autorität des Komponisten und auch die Fiktionalität der Bühnenfiguren.

Der Bühnen-Tschaikowsky entkommt dieser Bedrohung, indem er nicht etwa seinen Hermann umschreibt, sondern letztlich sich selbst ganz in sein Werk hineinschreibt: Er erfindet für sich die Opernrolle des Fürsten Jelezki (der in der Tat nur in der Oper und nicht in Puschkins literarischer Vorlage vorkommt) und erschafft damit einen gesellschaftlich arrivierten und in jeder Hinsicht unantastbaren Antagonisten, der am Ende als einzige zentrale Figur überleben wird. Damit verhandelt der Bühnen-Tschaikowsky in seiner Oper auch das Problem des Todes: Dadurch, dass der Bühnen-Tschaikowsky Teil seines Kunstwerks wird, wird er mit diesem gewissermaßen unsterblich.

Daran knüpfen sich freilich neue Probleme. Durch die Gleichsetzung des schwulen Bühnen-Tschaikowsky mit Lisas offiziellem, gesellschaftskonformem Bräutigam hat der Regisseur zwar seine Komponisten-Figur endlich fest im Werk verankert. Aber er zwingt seinem Bühnen-Tschaikowsky-Jelezki fortan auch eine geradezu schizophrene Rolle auf. Die beiden Personenanteile überlagern einander;

tism of the Director: Stefan Herheim's Production of Tchaikovsky's 'Queen of Spades «, in: Mitteilungen der Tschaikowsky-Cesellschaft 26 [2019], S. 31-43, S. 32): TThe libretto, too, has been thoroughly rethought; Cerman's words , You are old and have not long to lives, originally directed towards the countess, are here addressed to the figure of Tchaikovsky himself, making the opera a meditation on the composer's well attested sense of mortality." 
das Bild gerät ins Flackern, und mal überwiegt die eine, mal die andere Rolle. Konkret bedeutet das: Der Bühnen-Komponist, der seinen Hauptfiguren Musik auf den Leib schreibt und ihre verbotene Liebe anstachelt, tut dies, obwohl er zugleich der betrogene Liebende Jelezki ist, dessen große Gefühle für Lisa unerwidert bleiben. Die in dieser Bühnenrolle liegende, vollkommen plausible Abneigung Jelezkis gegenüber Hermann hindert den Bühnen-Komponisten freilich nicht daran, sich erneut in eine emotionale Beziehung zu seinem Helden zu begeben: Unmittelbar bevor Hermann der alten Gräfin das Geheimnis der drei gewinnbringenden Spielkarten abringen will und der Bühnen-Tschaikowsky ihn dabei in ein fatales Konglomerat aus Ängsten, Realitätsverlust, Zwangshandlungen und Wahnvorstellungen hineinkomponiert (2. Akt, 4. Bild der Oper), kommt es vor dem Jugendbild der alten Gräfin (bzw. vor dem Porträt der historischen Nadeshda von Meck) zu einem Déjà-vu. Der Bühnenraum zeigt erneut die Ausgangssituation des Opernabends: das Bild, den Stuhl, in dem Hermann nun kraft- und willenlos zusammenbricht, und Tschaikowsky, der ihm die Hand küsst - diesmal ohne brüske Zurückweisung. Neu ist in dieser Szene das Glas Wasser, das auf dem Tisch steht und unübersehbar den Tod in diese Denkfigur einführt - und zwar nicht als Hinweis auf den bevorstehenden Tod der alten Gräfin, sondern auf die Todesgefahr, in die sich der Bühnen-Komponist durch seine Gefühle für Hermann ${ }^{24}$ bzw. für seinen bezahlten Liebhaber begibt. In demselben Sinne verweist auch die Uhr auf dem Kaminsims, die auf Zwölf steht, auf den Tod.

Die Rettung erfolgt, indem Herheims Bühnen-Tschaikowsky abermals in die Rolle des Fürsten Jelezki schlüpft und Hermann im finalen Kartenduell gegenübertritt. Doch sogar in dieser Konstellation flackern die Rollenbilder, ist doch auch diese Szene zugleich eine kreative Erfindung des Bühnen-Komponisten: Unmittelbar bevor der Bühnen-Tschaikowsky zu Jelezki wird, schiebt er Hermann nachdrücklich zum Flügel; das Kompositionsinstrument wird zum Tisch für das Glücksspiel, die Notenblätter repräsentieren die Spielkarten. Als Duell zwischen Notenblatt (Jelezki) und Pistole (Hermann) inszeniert, verliert Hermann gegen Jelezki. Als er sterbend in den Armen der Tschaikowskyschen Dreieinigkeit von Jelezki, BühnenKomponist und Ex-Liebhaber zusammenbricht, hat der Bühnen-Komponist die brutale Zurückweisung, die ihm im Vorspiel angetan wurde, komponierend ausgelöscht - freilich um den Preis, dass erwiderte Zuwendung nur im Augenblick des Todes möglich ist. Aber es ist der Tod Hermanns, nicht sein eigener.

In diesem Sinne muss also auch die Überlegung zu Tod und Unsterblichkeit präzisiert werden: Indem der Bühnen-Tschaikowsky in die Opernrolle des einzi-

24 Man beachte, dass Herheim in seinen beiden Inszenierungsvarianten die deutsche Namensform »Hermann« wählt (vgl. Anm. 3). Im Kontext seiner Inszenierung erhält die zweite Silbe «-mann« eine für die Beziehung zwischen Hermann und Tschaikowsky-jeletzki sehr spezifische Nebenbedeutung. 
gen Überlebenden schlüpft und den Tod Hermanns aktiv herbeikomponiert, löst er für sich selbst die drohende Verknüpfung von Homosexualität und Tod auf und überlebt - zumindest als Opernfigur Jelezki. Sein Überleben ist dabei an den Tod des Geliebten und an das Ende jeder Liebesmöglichkeit gebunden.

Freilich wäre Herheim nicht Herheim, wenn er diese Beobachtung schlussendlich nicht ebenfalls wieder ins Flackern bringen und sie damit als primär theatrales Element einer Theaterwirklichkeit decouvrieren würde. Denn als die Gruppe der um Hermanns Leiche gedrängten Choristen von der Bühne abgeht, bleibt nur ein einziger Körper zurück: Es ist nicht Hermann, sondern der Bühnen-Tschaikowsky, der reglos auf der Erde liegt - wie schon einmal, am Ende der Ouvertüre, nachdem er demonstrativ das Glas Wasser ausgetrunken hatte und Lisa ihm in der Gestalt eines schwarzen Todesengels erschienen war. Damals kam er wieder zu sich, um seine Oper zu komponieren. Doch jetzt? Ist er jetzt tot? Ist sein Versuch, sein Schicksal komponierend umzuschreiben, gescheitert? Oder hat er seine gesamte Oper vielleicht nur geträumt? Wieder schiebt Herheim mehrere Bedeutungsebenen übereinander, und das Publikum muss selbst entscheiden, welchem der Bilder es den Vorzug gibt und wie sehr es in dem Vexierspiel der Deutungsangebote das magische Universum des Theaters als Theater erkennen möchte.

Ein solches Erzählen auf mehreren Ebenen setzt gegen die anfänglich so platt wirkende Eindeutigkeit des Bühnen-Tschaikowsky eine Kunst der Ambivalenzen und eine Vieldeutigkeit, die weit mehr ist als nur der Versuch, ausgewählte Eckpunkte einer bekannten Komponisten-Vita auf die Bühne zu bringen. Statt Tschaikowskys vielleicht emotionalstem und dramatischstem Bühnenwerk eine autobiographische Deutung aufzuzwingen, wird der Bühnen-Tschaikowsky bei Herheim zum Sinnbild des großen romantischen Künstler-Genies, dem beim Komponieren Kunst und Leben unauflöslich ineinanderfließen. $\mathrm{Zu}$ diesem Topos gehört auch, dass seine menschliche Unvollkommenheit und seine scheiternde Liebe ihm genau jenes Leid verschaffen, das es ihm ermöglicht, gewissermaßen kathartisch sein >Meisterwerk zu schreiben und damit nicht als Opernfigur, sondern als Komponist tatsächlich unsterblich zu werden. Darüber hinaus erzählt Herheim eine Geschichte über das Ringen mit Homosexualität als Antrieb für Kunst und er zeigt außerdem, dass all das ein mit den Mitteln des Theaters hergestelltes Stereotyp ist, das (vielleicht nur zufällig) an Tschaikowsky festgemacht wird. Und so schwebt über allem die unsichtbare Mahnung, die durch René Magrittes Bildtitel ikonisch geworden ist: „Ceci n'est pas une pipe« - oder besser: Ceci n'est pas Tchaïkovski. Was wir hier sehen, zu erkennen meinen und zu verstehen glauben, sieht zwar auf den ersten Blick aus wie Tschaikowsky, aber: Das ist nicht Tschaikowsky!

... Schön wäre es, wenn die Untersuchung von Herheims Regiekonzept hier enden könnte. Aber das theatrale Verwirrspiel erstreckt sich nicht allein auf das bislang untersuchte Bühnenspiel. Schon vorher, in demselben Augenblick, in dem alle Lichter im Saal erlöschen und damit der Beginn der Londoner Aufführung signali- 
siert wird, lässt der Regisseur auf dem Bühnenvorhang ein Statement einblenden, das alle Ambivalenzen noch vor Vorstellungsbeginn anscheinend radikal zurücknimmt:

»Pyotr Il'yich [sic!] Tchaikovsky died as Russia's most celebrated composer in 1893 - officially of cholera.

Supposedly, he drank a glass of contaminated water.

Years before, he had married in a vain attempt to sredeem his tormented souk from his homosexuality. ${ }^{25}$

Es folgt ein Zitat des Komponisten:

»Only music can save me.

Its beauty reconciles us with life.«

Zerstört diese scheinbar wissenschaftlich abgesichert daherkommende Biographiebindung nicht jede Idee der Übertragbarkeit? Unter einer solchen Prämisse kann das Londoner Publikum Herheims Bühnen-Tschaikowsky kaum noch anders deuten als exakt so, wie Herheim es in der im Internet abrufbaren Einführungsveranstaltung vor der Premiere selbst formuliert: »He was a miserable human being who [...] probably killed himself. ${ }^{26}$ Die komplexere Idee von »Musikgeschichtstheater ${ }^{27}$ wird zur optisch visualisierten Komponistenbiographie simplifiziert, reduziert zugunsten einer kurzschlüssigen Gleichsetzung von Homosexualität, Tod und hochemotionaler Musik. Gegen die aktuelle musikwissenschaftliche Tschaikowsky-Forschung und ihr Bemühen um ein möglichst weites Spektrum an Quellen $^{28}$ und deren facettenreiche Deutungsmöglichkeiten setzt diese extrem verkürzende Wahrnehmung des historischen Komponisten das banale Klischee eines allein aus seiner Sexualität heraus erklärbaren Künstlers und eines allein aus der Sexualität des Künstlers heraus erklärbaren Opernwerks.

25 Die drei Textabschnitte werden nacheinander eingeblendet, lassen also Raum für Assoziationen und für Verknüpfungen mit eigenem Hintergrundwissen, sodass sie mottoartig die Wahrnehmung des Bühnen-Tschaikowskys mitprägen und sie sogar in eine klare Richtung drängen. In der Amsterdamer Inszenierung fehlt dieser Hinweis.

26 Royal Opera House: »Insights into The Royal Opera's Queen of Spades«, in:

YouTube, https://www.youtube.com/watch?v=AW6aFBzkkYo (aufgezeichnet am 18.12.2018, abgerufen am 06.09.2019) bei 1:04:13.

27 Vgl. Anna Langenbruch: »Wenn Geschichte klingt. Musikgeschichte auf der Bühne als geschichtstheoretischer Impuls«, in: dies. (Hg.): Klang als Geschichtsmedium. Perspektiven für eine auditive Geschichtsschreibung (Musikgeschichte auf der Bühne 1), Bielefeld 2018, S. 73-98.

Dokumentiert und auch bibliographisch erfasst auf Tschaikowsky-Cesellschaft e. V., https://w ww.tschaikowsky-gesellschaft.de/ (abgerufen am 06.09.2019) und Tchaikovsky Research, https ://www.tchaikovsky-research.net/ (abgerufen am 06.09.2019). 
Aber »Der Mensch ist immer mehr als er von sich weiß ${ }^{29}$, sagt der Philosoph Karl Jaspers. In diesem Sinne scheint es, dass auch die Kunst Herheims mehr ist, als der Regisseur von ihr weiß (oder von ihr preisgibt). Wenn sich Herheims minutiös durchgefeilte Bildersprache als ein Geflecht simultan überlagernder und wechselweise in den Vordergrund tretender Bedeutungszuweisungen darstellt, die sich an den drei rezeptionsgeschichtlichen Eckpunkten >Homosexualität<, >Tod >künstlerisches Schaffen festmachen - dann ist vielleicht auch Herheims verbales Deutungsangebot in der Einführungsveranstaltung nur ein weiterer Bestandteil seines grundsätzlichen Konzepts changierender Interpretationen: Die Wortkommentare des Regisseurs überlappen sich mit der Einsicht, dass das geschichtliche Individuum Tschaikowsky zwar tot ist, dass seine Musik aber unsterblich weiterlebt.

In diesem Zusammenhang erfährt die für das Konzept von >Musikgeschichtstheater nicht unerhebliche Frage, ob zur historischen Anbindung eines »Musikgeschichtstheaterwerks« auch originale Musik zitiert wird, eine sehr eigentümliche Antwort: Dadurch, dass in der Londoner Inszenierung von Tschaikowskys Oper Pique Dame nicht Tschaikowskys gedruckte Partitur das >Musikgeschichtstheater ist, sondern das, was der Regisseur dramaturgisch, visuell und erzählend daraus macht, erhält die gesamte erklingende Musik die Anmutung eines unterlegten Authentizitätssignals und wird damit gewissermaßen zu einem klingenden Kommentar, den zu dechiffrieren, zu deuten und zu verstehen dem Publikum ans Herz gelegt wird. Auf diesem Weg kann die vermeintlich allbekannte originale Partitur in ihrer Emotionalität und ihrer Authentizität - und auch in ihrem Bezug auf den historischen Komponisten Tschaikowsky - neu wahrgenommen und gewichtet werden: Erst durch Herheims Inszenierung entsteht hier ein >Musikgeschichtstheaterstück , in dem das Publikum die Musik nun gewissermaßen ganz neu erleben kann. - Ganz in diesem Sinne postuliert Herheim am Ende der Londoner Pique-Dame-Matinee: »That's, I guess, my highest ideal about doing this: to become one, actually with your own time and ... and people around you, through music and through listening - learning to listen, actually deeper than we are used to and forced to in our reality. «" Die grundlegende Konstante der Inszenierung - und aller Tschaikowsky-Inszenierungen - ist also die Musik, die hinter allen neu gefundenen Bildwelten und Bedeutungszuweisungen immer wieder in ihrer Innovationskraft, ihrer Einzigartigkeit und ihrer Frische neu erlebbar wird. Fern von Pseudobiographik und Künstlermythen legt Herheims Pique-Dame-Aufführung dem Tschaikowsky-Publikum genau dies ans Herz: »Learning to listen, actually deeper than we are used to and forced to in our reality«!

29 So die oft referierte Version; bei Karl Jaspers (Kleine Schule des Philosophischen Denkens, München 1965, S. 66) heißt es: »der Mensch [ist] grundsätzlich mehr als er von sich wissen kann«. 


\section{Quellen und Literaturverzeichnis}

\section{Quellen}

Petr Il'ič Čajkovskij: Polnoe sobranie sočninij [Gesamtausgabe], Bd. 9: Pikovaja Dama, Partitur, hg. von Anatolij Dmitriev, Moskau 1950.

Petr Il'ič Čajkovskij: Polnoe sobranie sočninij [Gesamtausgabe], Bd. 15b: Briefe 1890, hg. von Ksenija Davydova und Galina Labutina, Moskau 1977.

D. I.: [Kommentar zur Aufführung von Herheims Inszenierung am Royal Opera House London] am 22.01.2019, in: Your Reaction: What Did You Think of Tchaikovsky's >The Queen of Spadesı? Audience Responses and Press Reviews of Stefan Herheim's Staging of Tchaikovsky's Ambitious Opera, hg. von Mel Spencer, in: Royal Opera House London: Premierenseite $\mathrm{zu} » T$ The Queen of Spades«, Abschnitt »Blog«, https://www.roh.org.uk/news/your-reaction-what-did-you-think-of-t chaikovskys-the-queen-of-spades (abgerufen am 06.09.2019).

An American in Paris, Regie: Vincente Minelli, USA 1951.

P. [Aleksandr Sergeevič Puškin]: »Pikovaja dama«, in: Biblioteka dlja čtenija [Lesebibliothek], 2 (1834), S. 109-140.

Royal Opera House: "Insights into The Royal Opera's Queen of Spades", in: YouTube, https://www.youtube.com/watch?v=AW6aFBzkkYo (aufgezeichnet am 18.12.2018, abgerufen am 06.09.2019).

Pyotr Il'yich Tchaikovsky: The Queen of Spades. In ausgewählten Kinos ausgestrahl-

ter Premierenmitschnitt des Royal Opera House London, Aufführung am 22.01.2019. Stefan Herheim (Regie), Philipp Fürhofer (Bühnenbild, Kostüme), Bernd Purkrabek (Licht), Alexander Meier-Dörzenbach (Dramaturgie); Sergey Poliakov (Hermann), Vladimir Stoyanov (Jelezki/Tschaikowsky).

Pyotr Ilyich Tchaikovsky: Pique Dame, Regie: Stefan Herheim, De Nationale Opera, Amsterdam 09.06.-03.07.2016, DVD, Berlin: C Major Entertainment GmbH, 2018.

Peter Tschaikowski: Die Tagebücher, hg. von Ernst Kuhn, übers. von Hans-Joachim Grimm, Berlin 1992.

\section{Literatur}

Philip Ross Bullock: »The Authority of the Author and the Despotism of the Director: Stefan Herheim's Production of Tchaikovsky's >Queen of Spades'«, in: Mitteilungen der Tschaikowsky-Gesellschaft 26 (2019), S. 31-43.

Kadja Grönke: Čajkovskijs Puškin-Opern. Aspekte einer Werke-Einheit (ČajkovskijStudien 5), Mainz u.a. 2002. 
Kadja Grönke: »Čajkovskijs Tod - ein kritischer Literaturbericht «, in: Thomas Kohlhase (Hg.): Čajkovskijs Homosexualität und sein Tod: Legenden und Wirklichkeit, sowie weitere Beiträge anderer Autoren (Čajkovskij-Studien 3), Mainz u.a. 1998, S. 379403.

Karl Jaspers: Kleine Schule des Philosophischen Denkens, München 1965.

Anna Langenbruch: »Wenn Geschichte klingt. Musikgeschichte auf der Bühne als geschichtstheoretischer Impuls«, in: dies. (Hg.): Klang als Geschichtsmedium. Perspektiven für eine auditive Geschichtsschreibung (Musikgeschichte auf der Bühne 1), Bielefeld 2018, S. 73-98.

Alexander Poznansky: Tchaikovsky: The Quest for the Inner Man, New York u.a. 1991. Alexander Poznansky: „Čajkovskijs Homosexualität und sein Tod. Legenden und Wirklichkeit«, in: Thomas Kohlhase (Hg.): Čajkovskijs Homosexualität und sein Tod: Legenden und Wirklichkeit, sowie weitere Beiträge anderer Autoren (ČajkovskijStudien 3), Mainz u.a. 1998, S. 9-135.

Valerij Sokolov: »Briefe P. I. Čajkovskijs ohne Kürzungen: Unbekannte Seiten seiner Korrespondenz«, in: Thomas Kohlhase (Hg.): Čajkovskijs Homosexualität und sein Tod: Legenden und Wirklichkeit, sowie weitere Beiträge anderer Autoren (ČajkovskijStudien 3), Mainz u.a. 1998, S. 137-162.

Tchaikovsky Research, https://www.tchaikovsky-research.net/ (abgerufen am 06.09.2019).

Tschaikowsky-Gesellschaft e. V., https://www.tschaikowsky-gesellschaft.de/ (abgerufen am 06.09.2019).

Irene Ulrich: »Der heilige Sebastian: vom christlichen Märtyrer zur homosexuellen Utopie«, in: Elke Pahud de Mortanges und Franziska Metzger (Hg.): Orte und Räume des Religiösen im 19.-21. Jahrhundert, Paderborn 2016, S. 207-222. 